\title{
NÚMERO CROMOSÓMICO DE UNA POBLACIÓN DE ALSTROEMERIA PELEGRINA L. (ALSTROEMERIACEAE), ESPECIE ENDÉMICA DE CHILE
}

\author{
CHROMOSOMAL NUMBER OF A POPULATION OF ALSTROEMERIA \\ PELEGRINA L. (ALSTROEMERIACEAE), ENDEMIC SPECIES OF CHILE
}

\author{
Carlos Baeza ${ }^{1 *}$, Pedro Carrasco ${ }^{1}$, y Nicolás Villalobos ${ }^{2}$ \\ ${ }^{1}$ Departamento de Botánica, Facultad de Ciencias Naturales y Oceanográficas, Universidad de \\ Concepción, Casilla 160-C, Concepción, Chile \\ ${ }^{2}$ Departamento de Producción Animal, Facultad de Agronomía, Universidad de Concepción, Vicente \\ Méndez 595, Chillán \\ * Autor para correspondencia E-mail: cbaeza@udec.cl
}

\section{RESUMEN}

Existen estudios citológicos previos de Alstroemeria pelegrina, especie endémica de Chile, pero en ninguno de ellos se menciona con claridad la localidad del material estudiado. Se analizó citotaxonómicamente una población de esta especie, proveniente de Los Molles, Región de Valparaíso. Se encontró que esta población presenta un $2 \mathrm{n}=2 \mathrm{x}=16$ cromosomas y una fórmula cariotípica haploide $3 \mathrm{~m}+1 \mathrm{sm}+2 \mathrm{st}+2 \mathrm{t}$. Al comparar los datos biométricos e índices de asimetría del cariotipo de esta población con los datos publicados anteriormente, se concluye que efectivamente se trata de la misma especie.

Palabras clave: Alstroemeria, cromosomas, fórmula cariotípica, Los Molles, Chile.

\section{ABSTRACT}

There are previous cytological studies in Alstroemeria pelegrina, an endemic species of Chile, but none of them clearly indicates the location of the material studied. A population of Alstroemeria pelegrina, collected from Los Molles (Region of Valparaíso), was analyzed cytotaxonomically. It was found that this population presents a $2 \mathrm{n}=16$ chromosomes and a caryotypic haploid formula $3 \mathrm{~m}+$ $1 \mathrm{sm}+2 \mathrm{st}+2 \mathrm{t}$. By comparing the biometric data and asymmetry indices of the karyotype of this population with previously published data, it is concluded that it is definitely the same species.

Key words: Alstroemeria, chromosomes, caryotypic formula, Los Molles, Chile.

Recibido: 11 febrero 2020. $\quad$ Aceptado: 10 marzo 2020. 


\section{INTRODUCCIÓN}

La familia Alstroemeriaceae es exclusiva de Centro y Sudamérica (Muñoz y Moreira, 2003). En Chile, el género de mayor distribución y mayor número de especies es Alstroemeria. Este género está representado en nuestro país por ca. 56 taxones, incluidas variedades y subespecies (Finot et al., 2018). Una de las especies endémicas es Alstroemeria pelegrina L., quizás la más cultivada en el mundo, y considerada uno de los parentales primarios de todos los cultivares actuales (Stephens et al., 1993). Esta hermosa especie se distribuye en las regiones de Coquimbo y Valparaíso, muy restringida al litoral costero de esta área, ocupando roqueríos, acantilados y laderas de cerros.

Se trata de una hierba perenne con una inflorescencia con 1-3 rayos unifloros, con flores de 4,0-5,5 cm de largo, rosadas (Fig. 1 A); tépalos externos muy obovados, con el ápice con escotadura, con mucrón ancho y verdoso; los tépalos internos superiores son más angostos con una mancha amarilla en el medio, con rayitas rosadas, a veces púrpuras; tépalo inferior interno semejante a los superiores, algo más largo. Anteras purpureas. Fruto una cápsula de 1,8 cm de largo, con 6 costillas longitudinales. Semillas de ca. $3 \mathrm{~mm}$ de diámetro (Muñoz y Moreira, 2003; Finot et al., 2018).

Los estudios citotaxonómicos han resultado útiles en Alstroemeria, puesto que han permitido caracterizar y delimitar taxones, debido a la enorme estabilidad del cariotipo de cada especie y la casi nula variabilidad poblacional de ellos (Baeza et al., 2010). Estudios recientes en complejos taxonómicos han demostrado utilidad en el reconocimiento de taxones mediante diferencias en la estructura o índices de asimetría de los cromosomas (Cajas et al., 2009; Baeza y Ruiz, 2011; Baeza et al., 2006; 2010; 2015; 2016a; b; 2018; Negritto et al., 2015).

El objetivo de esta comunicación es entregar información de los cromosomas de una población de $A$. pelegrina proveniente de la región de Valparaíso, y comparar estos resultados con los indicados en la literatura, que no indican procedencia ni lugar de colección.

\section{MATERIALES Y MÉTODO}

Se analizó citotaxonómicamente una población de Alstroemeria pelegrina, proveniente de Chile, V Región de Valparaíso, Provincia de Petorca, Los

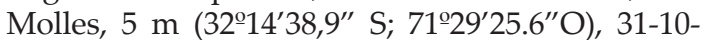
2018, recolectada por Nicolás Villalobos y Pedro Carrasco, número de colecta 1916, y depositada como voucher en el herbario CONC de la Universidad de Concepción, Chile.

El método de trabajo consistió en disectar puntas de raíces de 1-2 $\mathrm{cm}$ de longitud, a partir de material cultivado en invernadero, las cuales fueron cortadas y pretratadas con una solución de 8 -hidroxiquinolina $(2 \mathrm{mM})$ por $24 \mathrm{~h}$ a $5^{\circ} \mathrm{C}$. Luego, se fijaron en una mezcla de etanol/ácido acético (3:1) durante $24 \mathrm{~h}$. Se realizaron aplastados de puntas de raíces, previo una hidrólisis ácida con $\mathrm{HCl} 0,5 \mathrm{~N}$ por $17 \mathrm{~min}$ a $42^{\circ} \mathrm{C}$. Posteriormente, se lavó el material y se tiñeron las puntas de raíz con orceína al 1\%. Los cromosomas se fotografiaron en un microscopio con cámara de video incorporada (Zeiss Axioskp, Frankfurt, Alemania). Las placas

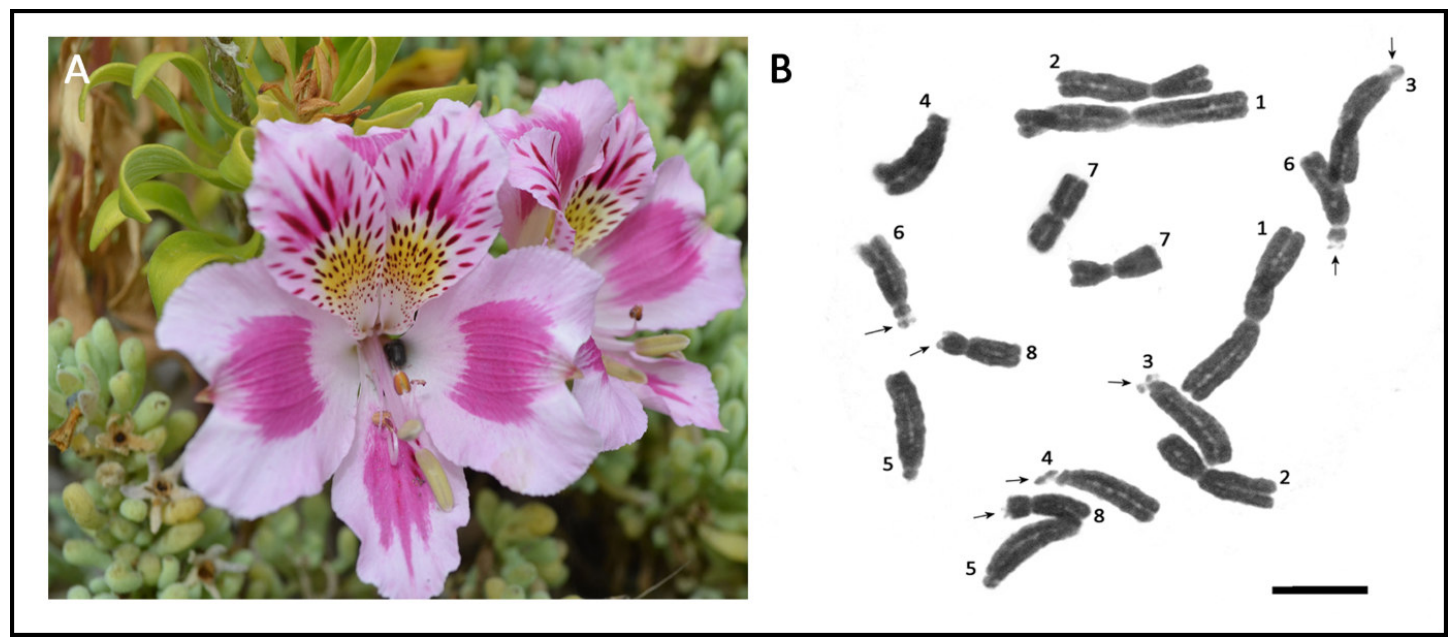

Fig 1. A: Flores de Alstroemeria pelegrina. B: Placa metafásica. Las flechas indican los homólogos con satélites. Escala $=\mathbf{1 0} \mu \mathrm{m}$.

Fig. 1. A: Flowers of Alstroemeria pelegrina. B: Methaphase plate. The arrows indicate the homologues with satelites. Scale $=10 \mu \mathrm{m}$. 
metafásicas fueron medidas con la ayuda del programa computacional MicroMeasure 3.3 (Reeves, 2001). A partir de 5 placas metafásicas se calcularon los índices de asimetría del cariotipo AsK\% (Arano y Zaito, 1980), TF\% (Huziwara, 1962) y Syi (Venora et al., 2002).

\section{RESULTADOS Y DISCUSIÓN}

Las cinco metafases estudiadas presentaron $2 \mathrm{n}=2 \mathrm{x}=16$ cromosomas, con una fórmula cariotípica haploide $3 m+1 s m+2 s t+2 t$, esto es, tres cromosomas metacéntricos, un cromosoma submetacéntrico con satélite en el brazo corto, dos cromosomas subtelocéntricos, ambos con satélites en el brazo corto, y dos cromosomas telocéntricos, uno de ellos con un satélite en el brazo corto (Tabla 1; Fig. 1 B). La Tabla 2 resume los datos de los índices de asimetría usados de la población analizada en este trabajo y se comparan con los datos obtenidos de Stephens et al. (1993).

Los resultados encontrados en esta población son coincidentes con los encontrados en los trabajos de Tsuchiya y Hang (1989), y Stephens et al. (1993). Todas las poblaciones presentan $2 \mathrm{n}$ = 16 cromosomas, la misma fórmula cariotípica haploide, y prácticamente los mismos valores en los índices de asimetría del cariotipo analizados, además de compartir una longitud total haploide de los cromosomas (LTC) muy similares (Tabla 2). La única diferencia encontrada es la presencia en este reporte de un satélite en el par cromosómico 4 , en cambio, en los trabajos anteriores es el par 5 el que presenta este satélite y no el 4 (Fig. 1 B, flechas).

\section{CONCLUSIÓN}

Se concluyó que las plantas analizadas por Tsuchiya y Hang (1989) y Stephens et al. (1993) corresponden efectivamente a A. pelegrina, aunque en sus reportes no indicaron con claridad la procedencia y la localidad del material.

Se reafirma nuevamente la gran estabilidad del cariotipo en poblaciones de Alstroemeria, lo que hace de la citotaxonomía una herramienta poderosa como fuente complementaria a la sistemática vegetal en este grupo de monocotiledóneas.

\section{AGRADECIMIENTOS}

Trabajo financiado por proyecto VRID № 217.111.063-1.0. También se agradece al Departamento de Botánica de la Universidad de Concepción todas las facilidades otorgadas para realizar este trabajo.

Tabla 1. Mediciones cromosómicas de Alstroemeria pelegrina L. Se detallan las longitudes promedio como porcentaje de la longitud del genoma haploide de 5 metafases.

Table 1. Chromosomal measurements of Alstroemeria pelegrina L. Mean length as percent of the haploid genome length of 5 metaphases is indicated.

\begin{tabular}{crrrccc}
\hline $\begin{array}{c}\text { Par } \\
\text { cromosómico }\end{array}$ & Brazo largo & \multicolumn{2}{c}{ Brazo corto Largo relativo } & Largo total & $\begin{array}{c}\text { Radio del } \\
\text { brazo (L/C) }\end{array}$ & $\begin{array}{c}\text { Tipo de } \\
\text { cromosoma* }\end{array}$ \\
\hline & $\%$ D.S. & $\%$ \% D.S. & $\%$ & $\mu \mathrm{m}$ & & \\
1 & $40,4 \pm 0,2$ & $38,9 \pm 0,1$ & 79,2 & 24,4 & 1,03 & $\mathrm{~m}$ \\
2 & $23,9 \pm 0,3$ & $16,6 \pm 0,2$ & 40,4 & 12,5 & 1,40 & $\mathrm{~m}$ \\
3 & $31,7 \pm 0,2$ & $4,2 \pm 0,2$ & 35,9 & 11,1 & 7,5 & $\mathrm{t}$ - sat \\
4 & $26,3 \pm 0,2$ & $4,6 \pm 0,3$ & 30,9 & 9,5 & 5,7 & $\mathrm{st}$ - sat \\
5 & $24,9 \pm 0,1$ & $3,3 \pm 0,2$ & 28,2 & 8,7 & 7,5 & $\mathrm{t}$ \\
6 & $22,9 \pm 0,1$ & $3,7 \pm 0,1$ & 26,6 & 8,2 & 6,2 & $\mathrm{st}$ - sat \\
7 & $12,2 \pm 0,2$ & $10,5 \pm 0,2$ & 22,7 & 7,0 & 1,2 & $\mathrm{~m}$ \\
8 & $14,9 \pm 0,1$ & $5,8 \pm 0,1$ & 20,7 & 6,4 & 2,6 & $\mathrm{sm}$ - sat \\
\hline
\end{tabular}

* m = metacéntrico; t-sat = telocéntrico con satélite; st = subtelocéntrico con satélite; sm = submetacéntrico con satélite.

Tabla 2. Comparación entre la población analizada y la reportada por Stephens et al. (1993). Table 2. Comparison between the population analyzed and that reported by Stephens et al. (1993).

\begin{tabular}{lcccc}
\hline Población & LTC $(\mu \mathrm{m}) \pm$ D.S. & AsK\% \pm D.S. & TF\% \pm D.S. & Syi \pm D.S. \\
\hline Stephens et al., 1993 & 90,8 & 70,6 & 28,4 & 40,4 \\
Población analizada & $87,8 \pm 6,4$ & $72,1 \pm 3,2$ & $27,9 \pm 2,8$ & $38,7 \pm 2,6$ \\
\hline
\end{tabular}




\section{LITERATURA CITADA}

Arano, H., and H. Saito. 1980. Cytological studies in family Umbelliferae 5. Karyotypes of seven species in subtribe Seselinae. Kromosomo 2:471-480.

Baeza, M., O. Schrader, E. Ruiz, and M. Negritto. 2006. Análisis comparativo del cariotipo en poblaciones de Alstroemeria ligtu subsp. ligtu y A. ligtu subsp. simsii (Alstroemeriaceae) de Chile. Darwiniana 44(2):313-318.

Baeza, C., E. Ruiz, and M. Negritto. 2010. Comparative karyotypic analysis in the Alstroemeria hookeri Lodd. (Alstroemeriaceae) complex sensu Bayer (1987). Genet. Mol. Biol. 33(1):119-124.

Baeza, C., and E. Ruiz. 2011. Alstroemeria hookeri Lodd. subsp. sansebastiana C.M. Baeza \& E. Ruiz, nueva para la flora de Chile. Gayana Bot. 68(2):313-315.

Baeza, C., L. Finot, and E. Ruiz. 2015. Comparative karyotype analysis of populations in the Alstroemeria presliana Herbert (Alstroemeriaceae) complex in Chile. Genet. Mol. Biol. 38(2):199-204.

Baeza, C., V. Finot, E. Ruiz, P. Carrasco, P. Novoa, and M. Rosas. 2016a. Análisis citotaxonómico del complejo Alstroemeria diluta E. Bayer (Alstroemeriaceae) de Chile. Gayana Bot. 73(2):221-227.

Baeza, C., V. Finot, E. Ruiz, P. Carrasco, P. Novoa, T. Stuessy, et al. 2016b. Comparative karyotypic analysis and cytotaxonomy in the Alstroemeria ligtu L. (Alstroemeriaceae) complex of Chile. Braz. J. Bot. 39(1):305-313.

Baeza, C., V. Finot, E. Ruiz, P. Carrasco, P. Novoa, M. Rosas, et al. 2018. Cytotaxonomic study of the Chilean endemic complex Alstroemeria magnifica Herb. (Alstroemeriaceae). Genet. Mol. Biol. 41(2):434-441.
Cajas, D., M. Baeza, E. Ruiz, and M. Negritto. 2009. Análisis citogenético en poblaciones de Alstroemeria hookeri Lodd. ssp. hookeri (Alstroemeriaceae) en la Región del Biobío, Chile. Gayana Bot. 66(2):117-126.

Finot, V., C. Baeza, and M. Muñoz-Schick, et al. 2018. Guía de campo de las Alstroemerias Chilenas. 295 p. Corma (eds), Concepción, Chile.

Huziwara, Y. 1962. Karyotype analysis in some genera of Compositae. VIII. Further studies on the chromosome of Aster. Amer. J. Bot. 49:116-119.

Muñoz, M., and A. Moreira. 2003. Alstroemerias de Chile. 140 p. Diversidad, distribución y conservación. Taller La Era, Santiago, Chile.

Negritto, M., C. Baeza, E. Ruiz, and P. Novoa. 2015. Alstroemeria marticorenae (Alstroemeriaceae), a new species from central Chile. Syst. Bot. 40:69-74.

Reeves, A. 2001. MicroMeasure: a new computer program for the collection and analysis of cytogenetic data. Genome 44:239-443.

Stephens, J., T. Tsuchiya, and H. Hughes. 1993. Chromosome studies in Alstroemeria pelegrina L. Int. J. Plant Science 154:565-571.

Tsuchiya, T., and A. Hang. 1989. Cytogenetics in the genus Alstroemeria. Herbertia 45:163-170.

Venora, G., S. Blangiforti, M. Ruffini, D. Pignone, F. Losavio, and R. Cremonini. 2002. Chromatin organisation and computer aided karyotyping of Triticum durum Desf. cv Timilia. Caryologia 55:91-98. 